\title{
Error Analysis and Amendment Technology of Vibration Wire Strain Sensors
}

\author{
Zhonglong $\mathrm{Li}^{1,2, \mathrm{a}}$, Xiaodi Zhu ${ }^{3, \mathrm{~b}}$, Zhenyu Liu ${ }^{1}$, Dejian $\mathrm{Xu}^{1}$
}

${ }^{1}$ School of Transportation Science and Engineering, Harbin Institute of Technology, Key Laboratory of traffic safety, specialty materials and intelligent control technology transportation industry Harbin 150090, China;

${ }^{2}$ Postdoctoral Station of Mechanics, Harbin Institute of Technology, Harbin 150090, China;

${ }^{3}$ The Communications Research Institute of Liaoning Province, Shenyang 110015, China

alizhonglong@hit.edu.cn or lizhonglong2004@126.com, ${ }^{\text {b }}$ hongjianggu@163.com

Keyword: Vibration Wire Strain Sensors, Error Analysis, Amendment Technology

\begin{abstract}
With good stability and high reliability, vibration wire strain sensors were extensively applied in many momentous projects. In the paper, the basic principle and working characters of vibration wire strain sensors were introduced. Also, error caused by working principle, structural bending deformation and temperature were analyzed. In the end, amendment technology was provided for improving measurement accuracy of structural strain.
\end{abstract}

\section{Introduction}

Vibration wire strain sensor is a kind of non-electric quantity measuring sensor, which is extensively applied in the world. It has advantages of simply structured, good durability, strong anti-interference ability, reliable and stable measured values, high accuracy and easy operation. Comparing with resistance strain sensor, it is more easily for data collection, transmission, processing and storage. Its usage may realize automatic tests with high accuracy. Therefore, vibration wire strain sensors are widely used in the environment of poor working conditions and high monitoring technical requirements such as civil engineering, harbor engineering, mechanical ship, reservoir, dam, etc. Now, it has turned to be an indispensable gauging tool for industry and scientific research. It is believed to have a great future of application and development.

In the paper, the working principle of vibration wire strain sensor is analyzed. Also, error analysis and amendment formulas through aspects of working principle structural bending deformation and temperature fields are discussed. Experiments were carried out to prove the correction of theoretical analysis.

\section{Working Principle}

Vibration wire strain sensor is mainly composed of diaphragm, vibration wire, clamp holder, vibrator and vibration pickup. The force requiring measurement will change the string tension through diaphragm. Then, vibrator will supply string excitation force to make the sting vibrate. Vibration pickup will convert the mechanical vibrations into the same frequency signal output. Therefore, the change of natural frequency is responded by change of electrical signal.

Based on vibration theory and relationship between string vibration and tension, the function of vibration frequency and structural strain may be obtained.

Relationship BetweenString Tension and Natural Frequence. Based on string vibration theory, considering the influence of string stiffness and ignoring its sag impact, the string differential equation of motion is obtained as following:

$E I \frac{\partial^{4} y}{\partial x^{4}}-T \frac{\partial^{2} y}{\partial x^{2}}+m \frac{\partial^{2} y}{\partial t^{2}}=0$

where $y$ is transverse vibration displacement (perpendicular to the string length), $x$ is vertical coordinates, $T$ is string tension increment caused by vibration, $E I$ is the flexural rigidity and $m$ is linear density. 
Similar to simply supported beam bearing axial force with hinges at both ends, the boundary condition is $y(0)=0, y^{\prime \prime}(0)=0, y(L)=0, y^{\prime \prime}(L)=0$ The equation of equivalent expressions is:

$\sin \alpha l=0$

where $\alpha^{4}=\frac{\omega^{2} m}{E I}$.

The solution of Eq. 2 is $\alpha_{n} l=n \pi(n=1,2,3, \cdots)$. The equation of string n-order natural is:

$\omega_{n}=\frac{n \pi}{l} \sqrt{\frac{T_{n}}{m}\left(1+\frac{n^{2} \pi^{2}}{l^{2}} \frac{E I}{T_{n}}\right)}$

The equation of string tension $T_{n}$ is:

$$
T_{n}=\frac{m \omega_{n}^{2} l^{2}}{n^{2} \pi^{2}}-E I\left(\frac{n \pi}{l}\right)^{2}
$$

The natural frequency of vibration is $\omega_{n}=\left(\frac{n \pi}{l}\right)^{2} \sqrt{\frac{E I}{m}}$. Using the relationship of $\omega=2 \pi f$, the function between string tension and natural frequency can be obtained as $T_{n}=A f_{n}^{2}$, where $A=\left(\frac{l^{2}}{n^{2}}-\pi^{2}\right) 4 m f_{n}^{2}, T_{n}=\left(\frac{l^{2}}{n^{2} \pi^{2}}-1\right) m \omega_{n}^{2}=\left(\frac{l^{2}}{n^{2}}-\pi^{2}\right) 4 m f_{n}^{2}$.

If ignoring the impact of string flexural rigidity, Eq. 3 can be changed as following:

$$
f_{n}=\frac{n}{2 l} \sqrt{\frac{T_{n}}{m}}
$$

Eq.5 reflects the relationship between natural frequency $f_{n}$ and string tension $T_{n}$.

Calculation Model.In fact, stress increment is what actual test really cares about. Therefore, if begin frequency is $f_{0}$ and the end frequency is $f_{n}$, the increment is $\Delta T_{n}=A\left(f_{n}^{2}-f_{0}^{2}\right)$. This equation can be used to calculate stress.

\section{Error Analysis and amendment Technology}

Each kind of sensor has its characteristics including using conditions and environment. The error reasons are analyzed with considering the characteristics of working principle, structural bending deformation and temperature field. Further more, the amendment function is provided.

Amendment Technology Based on Working Principle. Based on vibration theory, the proportional relationship between string tension and 2-order natural frequency is derived. This equation shows sensor has nonlinear characteristic. However, in practice, linear relationship is expected for convenience of calibration and data processing. Therefore, in this paper, natural frequency difference is used to amend the equation:

$$
\Delta T_{n}=A\left(f_{n}^{2}-f_{0}^{2}\right)+B\left(f_{n}-f_{0}\right)
$$

Where coefficient $A$ and $B$ can be obtained by least squares curve fitting.

\section{Amendment Technology Based on Structural Bending Deformation}

Because the theory of vibration wire strain sensor is based on string theory, it is accurate when only under axial tension or stress. However, this state is rare in test of real project. Generally, structures are under bending force, which means it is necessary to study application condition and amendment of vibration wire strain sensor based on structural bending deformation.

The element force state of structure under bending deformation is shown as Fig.1. The relationship between real test strain and theory one is shown as following: 


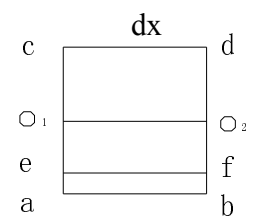

(a) before

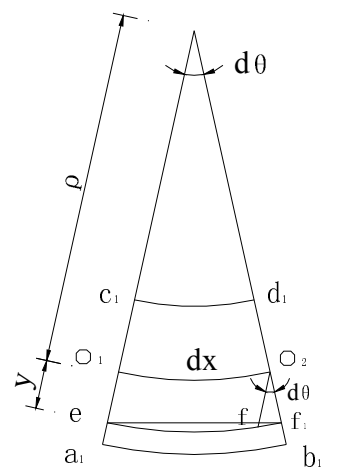

(b)after

Fig.1-1 Element deformation under bending deformation

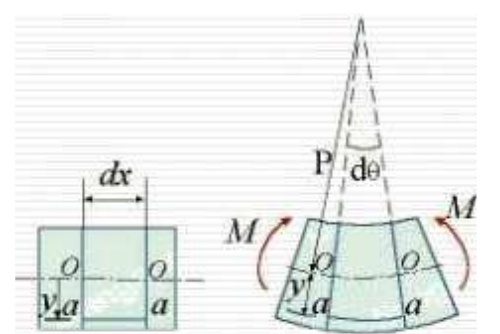

(a)

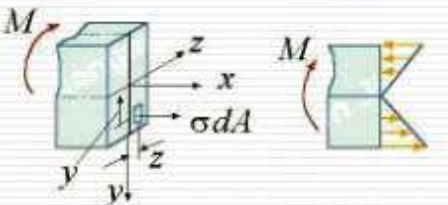

(c)

(d)

Fig.1-2 Element strain under bending

Theory of Strain. Based on mechanics of material, the theory value of strain can be obtained as following:

$$
\varepsilon_{l}=\frac{f \tilde{f_{1}}}{e f}=\frac{(\rho+y) d \theta-\rho d \theta}{\rho d \theta}=\frac{y}{\rho}
$$

which may be expressed shortly as : $\varepsilon=\frac{y}{\rho}$.

Real Test Value of Strain. During working, the output of vibration wire strain sensor is actually due to string tension to vary degrees of frequency difference. Therefore, what it measures actually is the increment of string-length instead of increment of the arc-length, which causes error. The equation of real test value of strain is

$\varepsilon_{c}=\frac{\Delta l}{l}=\frac{e f_{1}-e f}{e f}=\frac{x \rho+y) \sin (d \theta / 2)-\rho d \theta}{\rho d \theta}$

When $d \theta \in[0,0.5]$, the relative error of $\sin (d \theta)$ and $d \theta$ is lower than $5 \%$, which may consider $\sin (d \theta)=d \theta$. After equivalent transformation, the equation above is the same as the theory one. When $d \theta$ is bigger than $0.5 \mathrm{rad}$, real test value should be amended.

Amendment Technology. Using geometric relation $\varepsilon=y / \rho$, physical relation $\sigma=E \varepsilon$ and static equilibrium relation $M=E I_{z} / \rho$, the relationship between neutral layer structure curvature and bending moments may be expressed as $1 / \rho=M / E I_{z}$. In practice, $d x$ is standard distance of sensor. For a given sensor, $d x$ is a constant value. The theory value and test value are:

theory value $\varepsilon_{l}=\frac{y}{\rho}=\frac{M y}{E I_{z}}$

test value $\quad \varepsilon_{c}=\frac{2(\rho+y) \sin (d \theta / 2)-\rho d \theta}{\rho d \theta}=\frac{2\left(\frac{E I_{z}}{M}+y\right) \sin \left(\frac{M d x}{2 E I_{z}}\right)-d x}{d x}$.

Therefore, $\frac{\varepsilon_{l}}{\varepsilon_{c}}=k=\left(\frac{M y}{E I_{z}}\right) /\left(\frac{2\left(\frac{E I_{z}}{M}+y\right) \sin \left(\frac{M d x}{2 E I_{z}}\right)-d x}{d x}\right)=\left(\frac{M^{2} y}{2 E I_{z}\left[\left(E I_{z}+M y\right) \sin \left(\frac{M d x}{2 E I_{z}}\right)-M d x\right]}\right)$.

The parameters in the above equation are known, which means the test value may be amended. 


\section{Amendment Technology Based on Temperature Field}

When using vibration wire strain sensor to measure concrete structural strain, it is necessary to remove the section temperature strain of concrete and temperature deformation caused by test point.

Assuming both concrete section temperature and temperature of steel string of vibration wire strain sensor increase a same value of $\Delta t$, the test value of strain is made up of two parts: free expansion deformation of concrete structure and steel string deformation caused by temperature variations.

Assuming test value is $\varepsilon_{t}$, free expansion deformation of concrete structure is $\varepsilon_{\mathrm{h}}$, steel string deformation caused by temperature variations is $\varepsilon_{g}$, so:

$\varepsilon_{h}=\alpha_{h} \Delta t, \quad \varepsilon_{\mathrm{g}}=\alpha_{\mathrm{g}} \Delta t$

Where $\alpha_{h}$ and $\alpha_{\mathrm{g}}$ are respectively the coefficient of linear expansion of concrete and steel string. Then,

$\varepsilon_{\mathrm{t}}=\left(\varepsilon_{h}-\varepsilon_{\mathrm{g}}\right)=\left(\alpha_{h}-\alpha_{\mathrm{g}}\right) \Delta t$

When the temperature change of concrete section is uniform, change of test value is caused by difference of coefficient of linear expansion of concrete and steel string.

Therefore, when using vibration wire strain sensor to measure strain, it is important to pay attention to the difference of coefficient of linear expansion of concrete and steel string, which causes test error.

\section{Conclusion}

Each kind of sensor has its characteristics including using conditions and environment. It is necessary to ensure the respectively amendment technology based on vibration wire strain sensor's working principle, real force state and environment. In the paper, errors and respectively amendment technology are discussed. The amendment equation improves the accuracy of sensor in the measurement, becoming more accuracy to evaluate structural stress level and may be useful to scientific experiment and practical industry.

\section{Reference}

[1] F. Yu, N. Gupta. An Efficient Model for Improving Performance of Vibrating-Wire Instruments, J. Measurement, Vol.37 (2005) No.3, p.278-283.

[2] T.L. Bai, Z.L. Deng, J. Xie and F.P. Hu: Accurate Mathematical Model of Vibrating Wire Sensors and its Application, J. Rock Mechanics and Engineering, Vol. 52 (2005) No.24, p.5966-5969.

[3] R.S. Zhang and T.M. Wang: Mechanics of Materials (China Building Industry Press, China 1997).

[4] Y.L. Chui, Y.M. Liu and F. Yu: Resonant Sensor Theory Formula Correction Method and its Application, J. Test and Measurement Technology, Vol. 10(1996), No.1, p.17-22.

[5] T.L. Deng, S.L. Zhao, Z.Y. Zhao and S.M. Zhao: Research on High Accuracy Vibrating Wire senor, J. Journal of Shandong University of Science and Technology, Vol. 29(2010), No.1, p.52-56. 